\title{
Calibration of the nonlinear ring model at the Diamond Light Source
}

\author{
R. Bartolini, ${ }^{1,2}$ I. P. S. Martin, ${ }^{1}$ and G. Rehm ${ }^{1}$ \\ ${ }^{1}$ Diamond Light Source, Oxfordshire, OX11 ODE, United Kingdom \\ ${ }^{2}$ John Adams Institute, University of Oxford, OX1 3RH, United Kingdom \\ F. Schmidt \\ CERN, Geneva 23, Switzerland \\ (Received 23 December 2010; published 11 May 2011)
}

\begin{abstract}
Nonlinear beam dynamics plays a crucial role in defining the performance of a storage ring. The beam lifetime, the injection efficiency, and the dynamic and momentum apertures available to the beam are optimized during the design phase by a proper optimization of the linear lattice and of the distribution of sextupole families. The correct implementation of the design model, especially the nonlinear part, is a nontrivial accelerator physics task. Several parameters of the nonlinear dynamics can be used to compare the real machine with the model and eventually to correct the accelerator. Most of these parameters are extracted from the analysis of turn-by-turn data after the excitation of betatron oscillations of the particles in the ring. We present the experimental results of the campaign of measurements carried out at the Diamond storage ring to characterize the nonlinear beam dynamics. A combination of frequency map analysis with the detuning with momentum measurements has allowed for a precise calibration of the nonlinear model that can accurately reproduce the nonlinear beam dynamics in Diamond.
\end{abstract}

DOI: 10.1103/PhysRevSTAB.14.054003

PACS numbers: 05.45.-a, 29.20.db

\section{INTRODUCTION}

The Diamond storage ring has been operating for users since January 2007 [1]. The linear optics of the machine was successfully implemented during commissioning using the LOCO package [2]. The beta beating is routinely corrected to $1 \%$ peak to peak or less and the linear coupling can be reduced to less than $0.1 \%$ if required $[3,4]$.

A reliable modelization and control of the linear optics is a crucial prerequisite for progressing with the modelization of the nonlinear beam dynamics. Once a good control of the linear optics was achieved, a campaign of measurements was set up to understand the nonlinear dynamics of the beam in the storage ring. The operational drivers for this activity are the understanding and the improvement of the injection efficiency, the Touschek lifetime, and the beam losses around the ring. A necessary step of this investigation is the determination of a reliable model of the nonlinear beam dynamics: the correct implementation of the nonlinear ring model is crucial to maximizing the performance of a synchrotron light source.

The improvement of the nonlinear machine model is a long and complicated process: on the one hand, all available measured magnetic components of each individual quadrupole and sextupole have been introduced into the model; for the main dipole magnets we have the magnetic

Published by the American Physical Society under the terms of the Creative Commons Attribution 3.0 License. Further distribution of this work must maintain attribution to the author(s) and the published article's title, journal citation, and DOI. measurements of just one single prototype. On the other hand, we have gathered a large wealth of measurements of dynamical quantities that provide information about the nonlinear model of the storage ring. The strategy is to modify the model such that each of the measured dynamics parameters is reproduced. The aim is not to determine the real model, which would require a perfect knowledge of all elements of the machine, but rather to find an effective model which is in agreement with all measurements. Such an effective model can be used to better understand, predict, and control the effects of the nonlinearities of the system.

The dynamical quantities which characterize the nonlinear beam dynamics are the chromaticities, the detuning with amplitude, the dynamic aperture, the frequency maps, the resonance driving terms, and the Touschek lifetime. Earlier attempts at nonlinear ring model calibration were performed by using the resonance driving terms to fit individual sextupoles [5]. In this paper we present the results of a complementary approach to model calibration achieved by using nonlinear chromaticities, dynamic apertures, and frequency maps.

Diamond is equipped with the necessary beam instrumentation to perform an advanced investigation of nonlinear beam dynamics. Various means to excite betatron oscillations are available: pinger magnets which give oneturn kicks to the stored beam and striplines which provide a continuous resonant excitation (albeit at small amplitudes). A crucial component is a properly calibrated system of highly performing turn-by-turn beam position monitors (BPMs). In particular, a correct understanding of the frequency maps and of the resonant driving terms depends 
critically on the precise reconstruction of the betatron oscillation from the turn-by-turn BPM data. Significant improvements in the modeling were made after a series of unsuccessful comparisons pointed out a number of subtleties in the BPM response to the betatron oscillations which required careful consideration. As a result the BPM response to the betatron oscillations has been better understood and corrected, resulting in a largely improved BPM calibration which allowed the modeling effort to succeed.

In this paper we first describe the progress made in the calibration of the nonlinear model by fitting the detuning with momentum and with amplitude as provided by the frequency maps. Section II reviews the method and the experimental results obtained at the Diamond storage ring. In Sec. III we will review the issues concerning the correct reconstruction of the betatron oscillations from the turnby-turn signals acquired at all BPMs. Conclusions will be drawn in Sec. IV.

\section{MEASUREMENTS AND MODELING}

The most easily accessible dynamical quantities that characterize the nonlinear beam dynamics are the chromaticities and the detuning with amplitudes. At Diamond it is possible to measure the tune dependence with momentum deviation by varying the $\mathrm{rf}$ frequency of the machine. The relation between a change of the rf frequency to the momentum deviation is complicated by the higher order terms in the expansion of the momentum compaction factor with off-momentum deviation, nevertheless it was possible to explore a range of momentum deviations sufficiently large to allow the measurement of the higher order chromatic components. All the simulations were performed with a modified version of the code AT [6] with additional pass methods which include the exact treatment of the Hamiltonian of the betatron motion and fringe fields in dipole and quadrupoles. These pass methods have been benchmarked with the code PTC [7].

The dependence of the betatron tunes on the momentum deviation and on the amplitude was used as a target vector for a fit of the eight sextupole family values. This technique was first used in [8] to optimize numerically the detuning with momentum deviation in the design stage of the Diamond storage ring. In the present study we included in the target vector also the values of the betatron tunes as measured during the acquisition of the frequency map in order to include as much information as possible about the nonlinear dynamics. In this way the target vector has the following set of components: the betatron tunes at a selected number of off-momentum values $Q_{x}\left(\delta_{1}\right), \ldots Q_{x}\left(\delta_{m}\right)$ and analogous for the vertical tunes; then we append to the same vector the betatron tunes at a selected number of amplitudes in $x$ and $y Q_{x}\left[(x, y)_{1}\right], \ldots, Q_{x}\left[(x, y)_{n}\right]$, and analogous for the vertical tune. If we measure the betatron tunes for $m$ off-momentum values $d p / p=\delta$ and $n$ frequency map points, the target vector has $2 m \cdot 2 n$ components:

$$
\begin{aligned}
\bar{A}_{\text {target }}= & \left\{Q_{x}\left(\delta_{1}\right), \ldots, Q_{x}\left(\delta_{m}\right), Q_{y}\left(\delta_{1}\right), \ldots, Q_{y}\left(\delta_{m}\right) ;\right. \\
& Q_{x}\left[(x, y)_{1}\right], \ldots, Q_{x}\left[(x, y)_{n}\right], Q_{y}\left[(x, y)_{1}\right], \ldots, \\
& \left.Q_{y}\left[(x, y)_{n}\right]\right\} .
\end{aligned}
$$

Each component of this vector will depend on the particular distribution of sextupole strengths in the ring, therefore we can write

$$
\bar{A}_{\text {target }}=\bar{A}\left(S_{1}, \ldots, S_{N}\right),
$$

where $S_{k}$ is the strength of the $k$ th sextupole family. In this way we obtain a target vector that we can both measure on the real machine and compute with the model. The square difference between these two vectors,

$$
\chi^{2}=\sum_{j=1}^{M}\left[A_{\text {measured }}(j)-A_{\text {target }}(j)\right]^{2},
$$

gives us a measure of the discrepancy between our nonlinear ring model and the real machine. Since this difference depends on the strength of the sextupole families, it can be used to build a least square fit of the sextupole strengths by family that best reproduces the nonlinear beam dynamics of the ring. The generalization of this procedure to fit each individual sextupole will be the object of further investigations.

The number of entries in the target vector has to be defined via trial and error and is therefore likely to be machine dependent. The best results at Diamond were achieved using a target vector containing momentum deviations in the range $-2.1 \%$ to $3.8 \%$ and a grid of onmomentum particles from on axis to $10 \mathrm{~mm}$ in horizontal and $2.5 \mathrm{~mm}$ in the vertical plane, with the final target vector containing 342 components. The result of the initial matching on the detuning with momentum showed excellent agreement with the measured data, as shown in Fig. 1. However, the extent of the frequency map was not well reproduced mainly due to a poor reconstruction of the vertical detuning with amplitude as shown in the comparison of Figs. 2 and 3.

The resulting discrepancies between the frequency map after the fit and the measured frequency map suggested further extending the fit parameters to include the systematic multipolar field errors in the main dipoles. In particular, the comparison to the measured frequency map has allowed the identification of an excessive contribution to the normal octupole component in the main dipole in the model which is responsible for significant variation in the extension of the frequency map in the betatron tunes space. The normal octupole component used in the model comes from the measurement of a single prototype magnet and was initially assumed as a systematic error in all the dipoles. This assumption produced an unrealistically large 


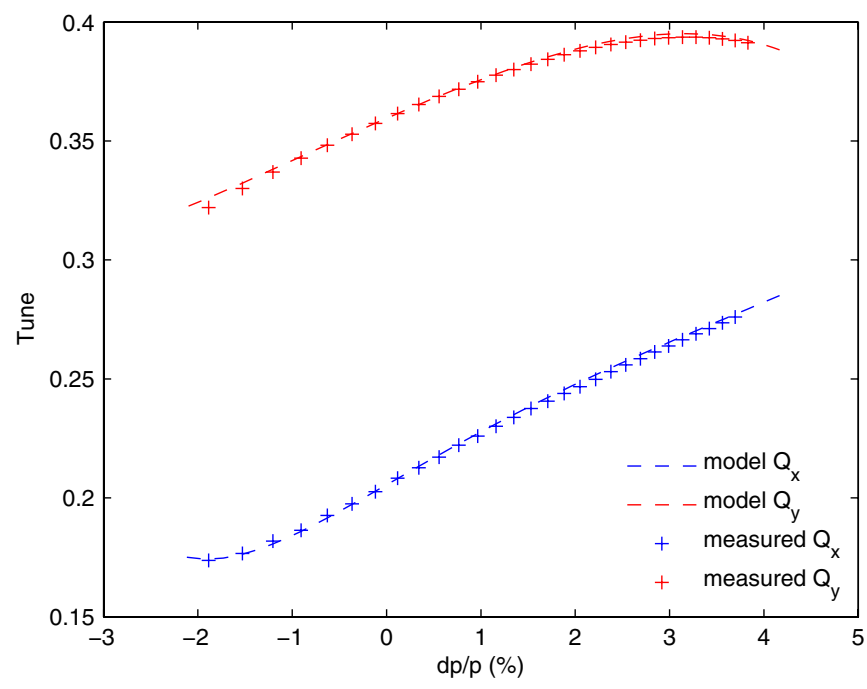

FIG. 1. Detuning with relative momentum deviation: comparison of measured to model data.

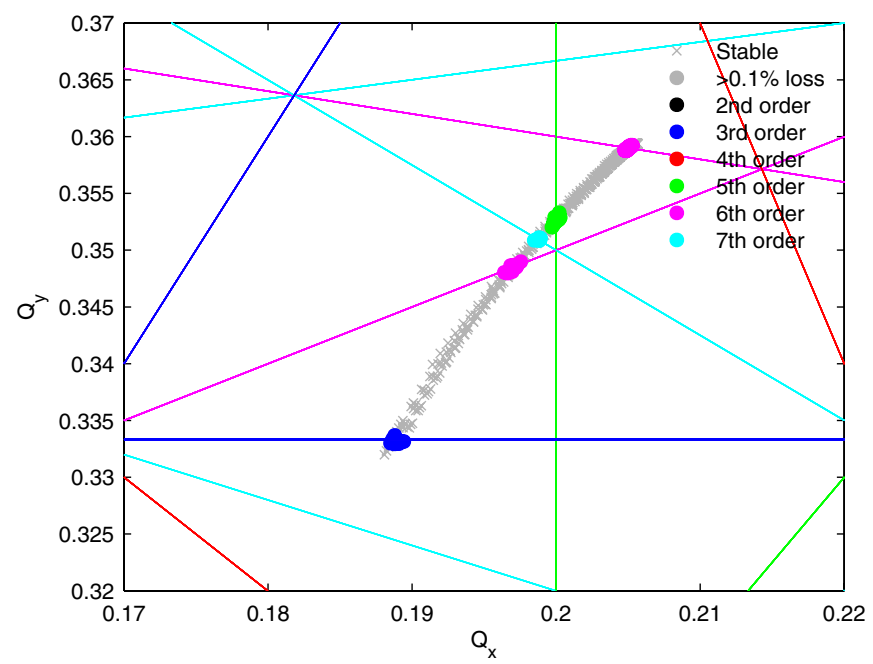

FIG. 2. Measured frequency map for the Diamond storage ring. The grey crosses identify betatron tune points corresponding to stable motion. The grey circles correspond to betatron tune points with losses larger than $0.1 \%$ of the beam after the kick. The colors identify the order of different resonances in the betatron tune space.

detuning with vertical amplitude and a wide frequency map. After extending the fit parameters to include the normal sextupole, octupole, and decapole components of the main dipole, we finally achieve a very good agreement of the frequency maps and measured dynamic aperture as shown in the comparison of Fig. 2 with Figs. 4 and 5. As a further independent check of the validity of this $6 \mathrm{D}$ model of the accelerator, we have compared the behavior of the synchrotron tune as a function of rf frequency for both model and measurement. Figure 6 shows the remarkable agreement achieved with the present modelization of the Diamond storage ring.

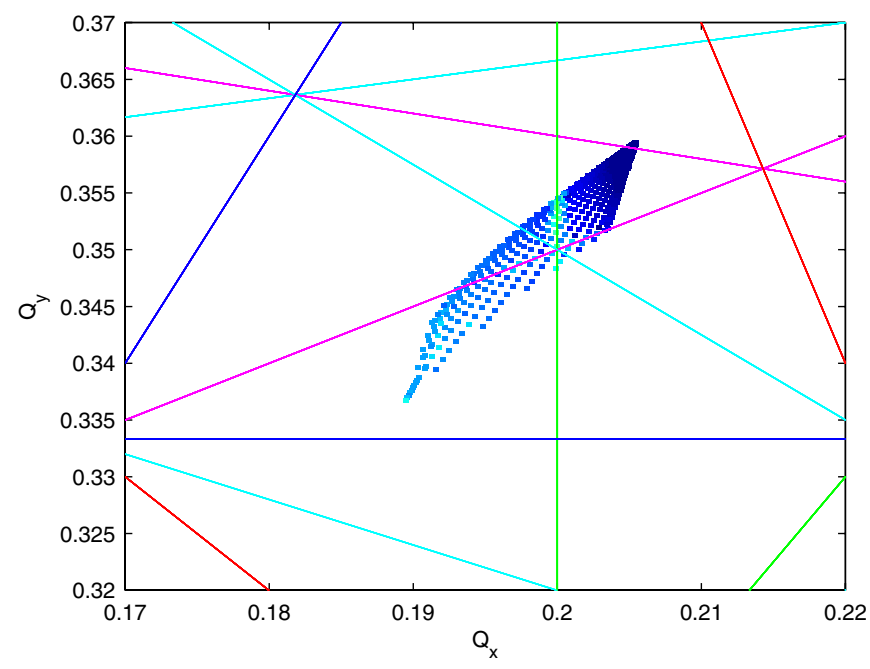

FIG. 3. Frequency map obtained after fitting only the sextupole gradients. See text for explanation. The color code corresponds to the diffusion coefficient of the betatron tunes, with dark blue indicating the most stable trajectories.

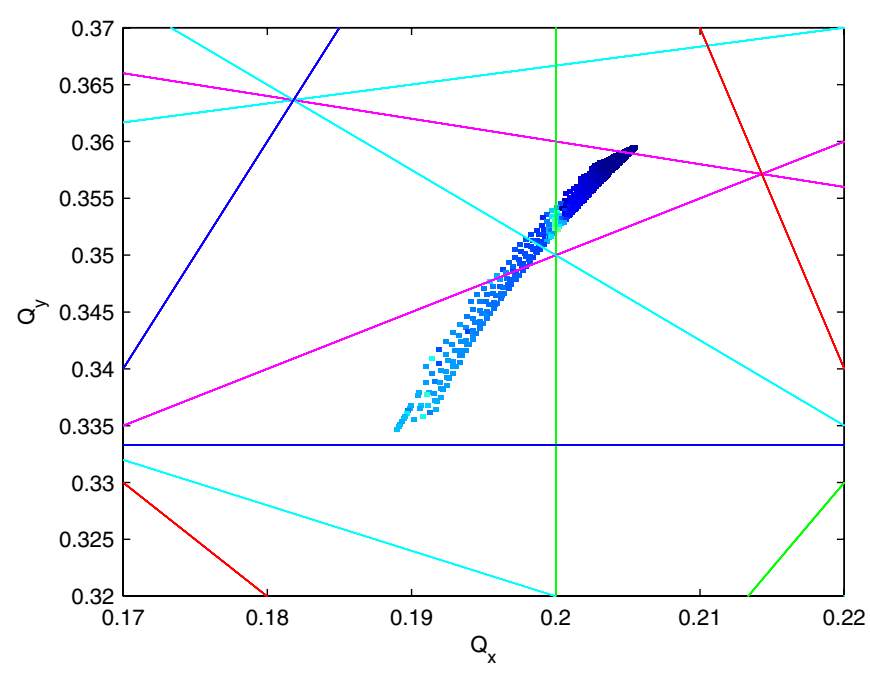

FIG. 4. Frequency map for the Diamond storage ring obtained from the model after including in the fit the systematic multipolar errors in the main dipoles. See text for explanation.

The variation of the sextupole gradients after the fit are within 3\% as reported in Table I. The values for the systematic multipole components in the main dipole are reported in Table II.

These values appear to be high with respect to the measurement errors of the sextupole gradients and a careful reanalysis of the calibration tables of the sextupole gradients is in progress to clarify the issue. However, we want to point out that the analogous analysis performed by LOCO predicts variation of the individual quadrupoles by more than $1 \%$ and they are in line with previous investigation of the nonlinear ring model based on the analysis of the resonant driving terms [5]. Another possible source of discrepancy is the lack of an equivalently good 

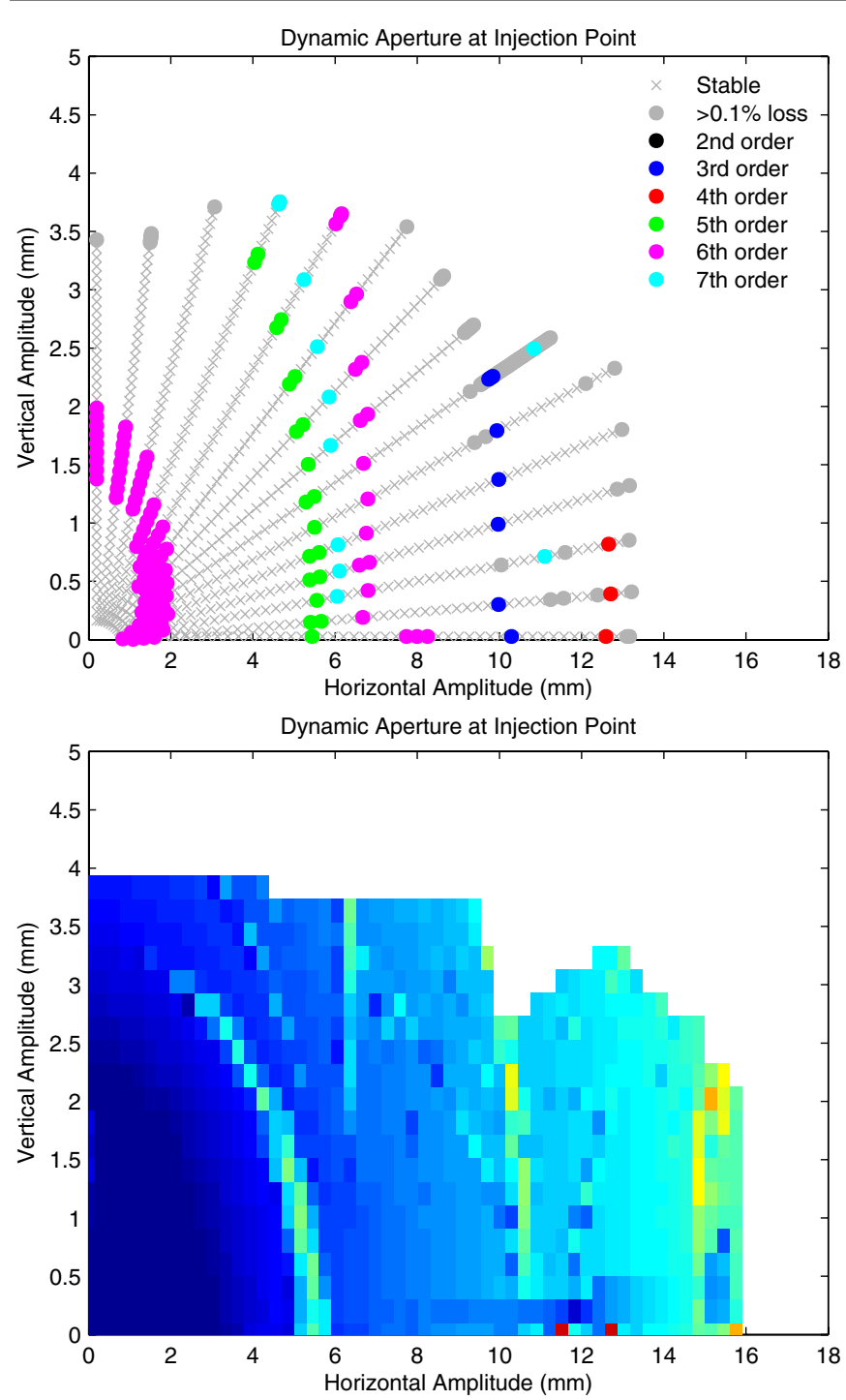

FIG. 5. Dynamic aperture and resonance lines plotted in the $(x, y)$ plane: measured (top) and model (bottom). The color coding of the points corresponds to that of Figs. 2 and 4.

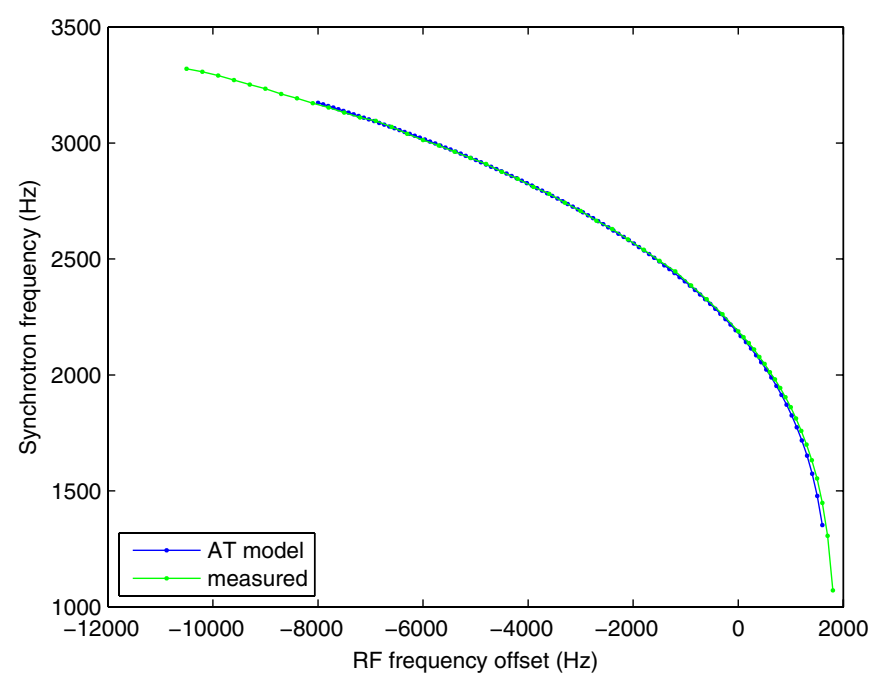

FIG. 6. Comparison of synchrotron frequency vs rf.
TABLE I. Relative variation of the sextupole strengths after the fit.

\begin{tabular}{lc}
\hline \hline Sextupole family & \% variation \\
\hline S1A & -1.5 \\
S2A & -1.4 \\
S1B & -3.4 \\
S2B & 0.4 \\
S1C & -0.2 \\
S2C & 0.3 \\
S1D & 3.2 \\
S2D & -1.3 \\
\hline \hline
\end{tabular}

TABLE II. Variation of the systematic multipolar components of the main dipole after the fit.

\begin{tabular}{lcr}
\hline \hline Multipole & Before fit & After fit \\
\hline Bend sextupole $\left(\mathrm{T} / \mathrm{m}^{2}\right)$ & -0.24 & -0.34 \\
Bend octupole $\left(\mathrm{T} / \mathrm{m}^{3}\right)$ & -4.63 & 0.12 \\
Bend decapole $\left(\mathrm{T} / \mathrm{m}^{4}\right)$ & -410.8 & -410.7 \\
\hline \hline
\end{tabular}

agreement with the natural chromaticity in the $V$ plane which is then redistributed among the gradients of the various sextupole families. The assessment of the effect of the sextupoles on the chromaticity in fact requires a proper understanding of the natural chromaticities. They were measured by shifting the main dipole field and using a Hall probe in the dipole magnet to measure the relative magnetic field variation. This produced a good agreement of the horizontal natural chromaticity $\left(\xi_{x 0} \sim-79\right)$ while still a few units were unaccounted for in the vertical natural chromaticity. We measured $\xi_{y 0} \sim-31$ while the ring model using hard edge dipoles gives $\xi_{y 0} \sim-33$. The hard edge model with the $1 /(1+d p / p)$ correction for the momentum dependence of the bending magnet vertical edge focusing [9] produces $\xi_{y 0} \sim-35$. The model which uses the exact Hamiltonian and dipole fringe field described with a symplectic integrator up to second order in the MAD-X PTC module [7,10] provides $\xi_{y 0} \sim-35$. In this last case the finite extent of the dipole fringe field was modeled with the product FINT*HGAP set to 0.03 to best match the longitudinal roll off of the dipole field. The quantities FINT, fringe field integral, and HGAP, magnet gap, specify the finite extent of the fringe fields as defined in [11] and, for the hard edge model, FINT*HGAP is zero. The extra linear focusing introduced by the finite extent fringe fields was compensated by refitting the main quadrupoles back to the desired tunes and optics functions. We found that varying FINT*HGAP does not produce a significant change in the natural chromaticity after refitting the linear optics. The origin of this discrepancy is still under investigation: it is believed that a more careful description of the magnetic field in the dipole is required and a more accurate measurement of the beam energy deviation using the resonant spin depolarization is under development. 


\section{BETATRON OSCILLATIONS FROM TURN-BY-TURN BPM DATA}

In order to correctly reconstruct the betatron oscillations from the turn-by-turn data signal recorded using our BPM system, a number of corrections to the beam data have to be applied.

First, we need to take into account how the BPM electronics produce the individual turn-by-turn data points. In our particular case, analog-to-digital converters (ADCs) are acquiring 220 samples per turn $(117 \mathrm{MHz}$ sampling rate) and this data stream is then filtered and decimated to produce precisely one position reading per turn. However, it is paramount to fulfill the Nyquist criterion in this decimation process, and so the filter has to span samples covering more than one turn; in fact, it is 1373 points long which equals 6.25 turns. As a result, turn-by-turn samples are not strictly independent, but will always be a linear combination of adjacent turns. Figure 7(a) shows the time filter $T$ (black dotted line) used for the construction of the turn-by-turn button signal $V$ from the ADC samples. The ADC samples are nonzero only in correspondence with the multibunch fill pattern which appears repeated over five turns (blue line). As noticed, the time filter covers an extent of about six turns and it is centered on top of the multibunch fill at the $n$th turn. In these conditions, the button signal $V$ at turn $n$ will be a linear combination of ADC samples, i.e.,

$$
V(n)=\sum_{m=1}^{6} \sum_{k=1}^{220} T[k+220 \cdot(m-1)] \cdot \operatorname{ADC}[(k+220) \cdot(n+m-1)] .
$$

As this process is entirely numerical as part of the digital signal processing in the BPM, it can be simulated for a given fill pattern and its alignment relative to the filter. In this way, an impulse and frequency response can be calculated. The impulse response $I$, given in Fig. 7(b), shows us how the button signal $V$ at a given turn $n$ is made of linear combinations of signals $U$ from different turns and allows casting the previous expression in a more compact form:

$$
V(n)=\sum_{m=1}^{6} I(m) \cdot U(n-m) .
$$

The discrete Fourier transform $I(\omega)$ of the impulse response $I(m)$ shows how the button response varies with frequency [see frequency response in Fig. 7(c)]. Furthermore, it is possible to calculate a reconstruction filter which will revert the effect of the convolution with the neighboring turns [Fig. 7(d)]: using basic properties of the discrete Fourier transform (DFT), the reconstruction filter is given by the inverse DFT of $B(\omega)=1 / I(\omega)$. Going back to the time domain, the reconstructed single turn button signal $U$ is expressed as a convolution, a) fill and filter (dotted)

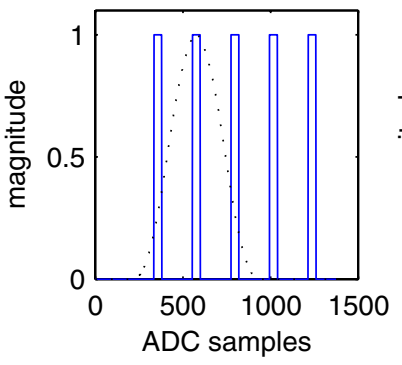

e) fill and filter (dotted)

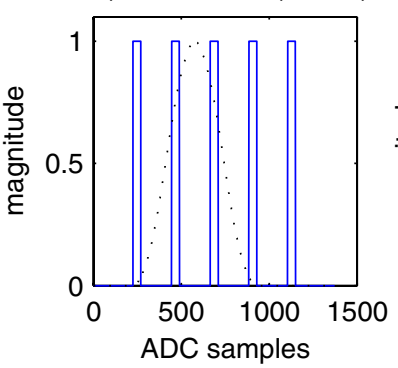

b) impulse response

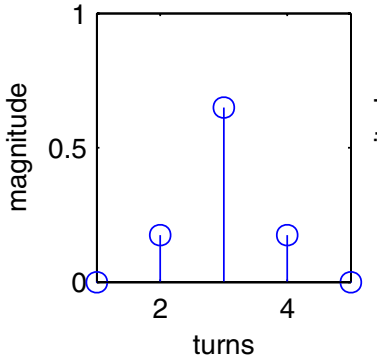

f) impulse response

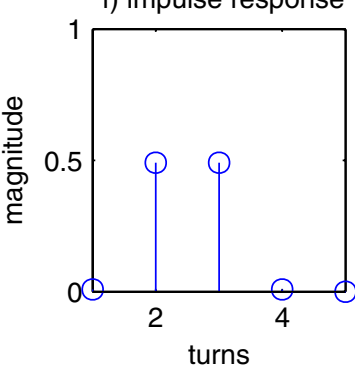

c) frequency response

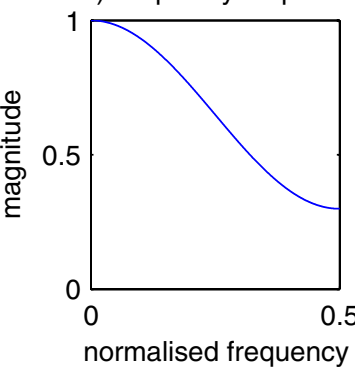

g) frequency response

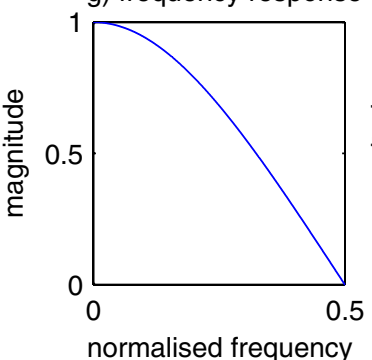

d) reconstruction filter

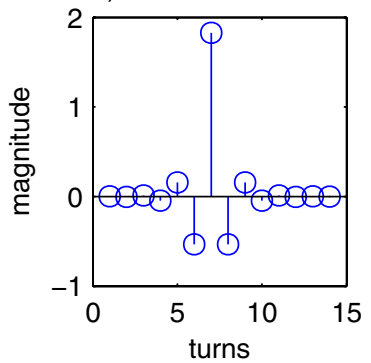

h) reconstruction filter

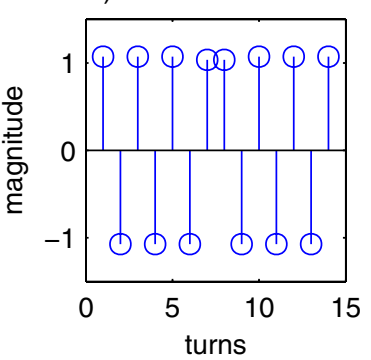

FIG. 7. Filtering effects in the BPMs and their removal using a reconstruction filter: fill pattern well aligned (top) and poorly aligned (bottom). See text for explanation. 


$$
U(n)=\sum_{m=1}^{6} B(m) \cdot V(n-m),
$$

which ultimately inverts relation (1) and removes the effect of the mixing of the signals from several neighboring turns.

In order for this reconstruction to be reliable, it is necessary to ensure that all BPMs around the ring take the turn-by-turn data synchronously and each applies the filter with a shift according to its location around the ring to take into account the time of flight. As can be seen from comparing the top graphs (a)-(d) and bottom graphs (e)_(h) in Fig. 7 it is also important to ensure the timing of the filter relative to the fill is such that a turn arrives central to the filter peak, as otherwise the reconstruction becomes poor or impossible.

Second, when investigating large amplitude oscillations, the geometric nonlinearities of the BPMs have to be taken into account as well. The standard method used to calculate the beam position from the individual BPM button responses is via the "difference over sum" mapping,

$$
\begin{aligned}
& X=K_{x}\left(\frac{(A+D)-(B+C)}{A+B+C+D}\right)-X_{0} \\
& Y=K_{y}\left(\frac{(A+B)-(C+D)}{A+B+C+D}\right)-Y_{0},
\end{aligned}
$$

where $A, B, C$, and $D$ are the button signals, $K_{x}$ and $K_{y}$ are the calibration factors, and $X_{0}$ and $Y_{0}$ are the offsets identified from beam-based alignment. As is well known, this mapping results in a poor estimation of the true beam position at large amplitudes [12]. To correct the geometric nonlinearity of the BPMs, we have implemented the correction strategy described in Ref. [12] to Diamond's four buttons BPM geometry [13]. In this method, the induced charge on each button is initially determined as a function of the simulated (true) beam position. This relationship between the charge on each of the four buttons and the true beam position $(x, y)$ can then be inverted, for example, using a Newton-Raphson method. In this way, if the relative charge induced on each button is known (i.e. the raw voltage signal is known for each button), the real beam position can be derived.

The final procedure used to reconstruct the measured amplitude of betatron oscillations from the raw BPM turnby-turn data is therefore to apply a deconvolution filter to the raw button signal, and then calculate the true beam position based on the method described. Figure 8 shows an example of reconstructed horizontal beam position as a function of pinger amplitude using (a) the difference over sum method, (b) applying the reconstruction filter to the difference over sum data, (c) calculating the beam position using the correction of the geometric nonlinearities on the unfiltered button signals, and (d) full reconstruction. In the example in Fig. 8 it is clear that the BPM data taken from the usual difference over sum method can significantly underestimate the actual position of the beam.

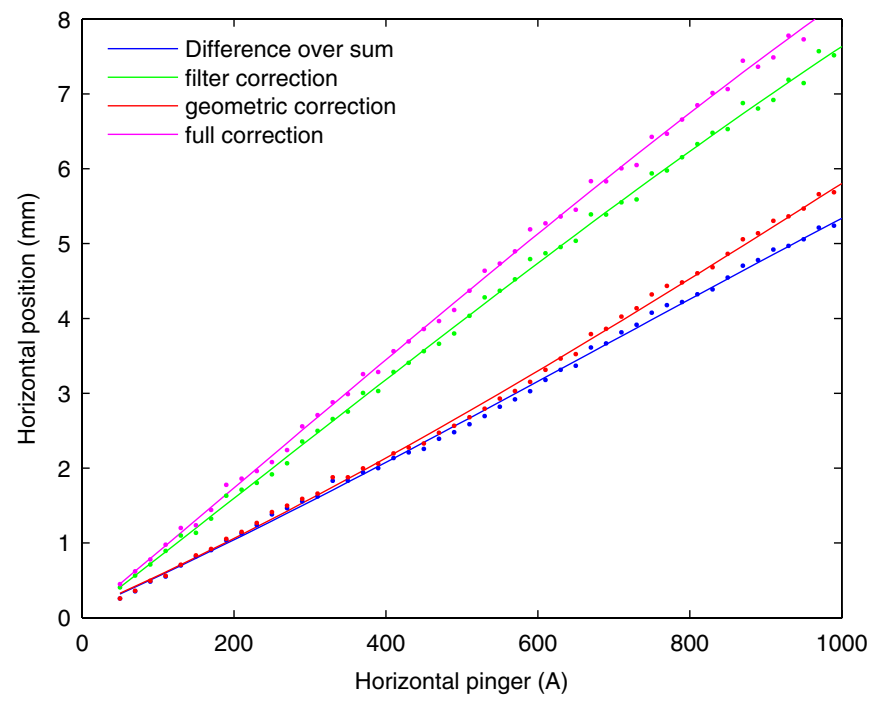

FIG. 8. Horizontal beam position as a function of pinger magnet strength, illustrating the relevance of correctly treating the individual button signals when reconstructing the measured position: blue are the raw BPM data given by the difference over sum formula; red data have the correction of the geometric nonlinearity only; green data have the deconvolution of the time filter only; magenta are the data with the full reconstruction. The solid lines are third order fit to the data.

We conclude that a successful fitting of the model against the measured detuning with momentum and amplitude data can only be achieved if both these aspects have been accounted for.

\section{CONCLUSION}

The latest result in the analysis of the nonlinear beam dynamics at the Diamond storage ring has been presented. The analysis of the nonlinear chromaticity and of the frequency map can provide very important information about the nonlinear model of the accelerator. We plan to provide an even better effective model of the nonlinear beam dynamics of the Diamond storage ring complementing the method presented with a more in-depth analysis which includes the resonance driving terms.

\section{ACKNOWLEDGMENTS}

We would like to thank P. Kuske (BESSY-II) and J. Rowland for help in the initial stages of this work.

[1] R.P. Walker, in Proceedings of the Asian Particle Accelerator Conference 2007 (RRCAT, Indore, India, 2007), p. 66.

[2] J. Safranek, Nucl. Instrum. Methods Phys. Res., Sect. A 388, 27 (1997).

[3] I. Martin et al., in Proceedings of the 11th European Particle Accelerator Conference, Genoa, 2008 (EPS-AG, Genoa, Italy, 2008), p. 3032. 
[4] C. Thomas et al., Phys. Rev. ST Accel. Beams 13, 022805 (2010).

[5] R. Bartolini et al., Phys. Rev. ST Accel. Beams 11, 104002 (2008).

[6] A. Terebilo, in Proceedings of the 19th Particle Accelerator Conference, Chicago, Illinois, 2001 (IEEE, Piscataway, NJ, 2001), p. 3203.

[7] F. Schmidt, in Proceedings of the 21st Particle Accelerator Conference, Knoxville, 2005 (IEEE, Piscataway, NJ, 2005), p. 1272.

[8] R. Bartolini et al., in Proceedings of the 10th European Particle Accelerator Conference, Edinburgh, Scotland, 2006 (EPS-AG, Edinburgh, Scotland, 2006), p. 2089.
[9] J. Bengtsson et al., in Proceedings of the 4th European Particle Accelerator Conference, London, UK, 1994 (EPS-AG, London, UK, 1994), p. 1021.

[10] E. Forest et al., http://frs.web.cern.ch/frs/report/fringe_ part_I.pdf (unpublished).

[11] H. Grote et al., CERN SL/90-13 (AP) (rev. 5); K.L. Brown, Technical Report No. SLAC-75, 1982.

[12] R. W. Helms et al., Phys. Rev. ST Accel. Beams 8, 062802 (2005).

[13] R. Bartolini et al., DLS Internal Report No. AP-SRREP0174, 2010. 